\title{
Research on The Characterization Methods of The Sealing Performance of The Bolted Flange Joints Under The Dynamic Loads
}

\author{
Ping $\mathrm{Wu}^{\mathrm{a}}$, Lanzhu Zhang ${ }^{\mathrm{b}^{*}}$ and Chaowen $\mathrm{Zhu}^{\mathrm{c}}$ \\ School of Mechanical and Power Engineering,East China University of Science and Technology, \\ Shanghai 200237, China.

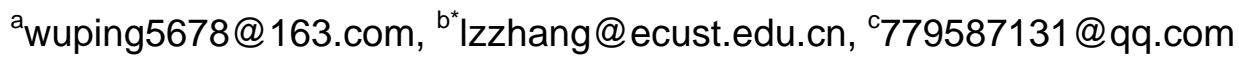 \\ Corresponding Author: Lanzhu Zhang
}

Keywords: Bolted Flange,Strain change,Acceleration,Correlation.

\begin{abstract}
In order to research the relationship between the change of bolt strain and the acceleration of pipe flange, in this paper, the vibration experiment platform which was a floating bolt flange pipe system under different bolt pre-tightening force was built. Experimental results show a high correlation between bolt strain and acceleration, and also show that the change of pipe acceleration can indirectly characterize the change of the flange sealing performance, which can provided the certain basis for online monitoring the sealing performance under certain special environments. Besides experimental results also indicate that the increase of pre-tightening force can improve the sealing performance of flange bolts in the case of vibration.
\end{abstract}

\section{Introduction}

In the petrochemical, nuclear and related industries, the sealing performance of bolted flange joint has an directly influence on the long-cycle operation of certain device. As the fast-increasing requirements on serving environments of bolted flange joints, particularly under the strong vibration conditions[1,2], the possibility of leakage due to the gasket sealing failure also grows, so the research on sealing performance of bolted flange joint under dynamic loading conditions is crucial.

In the bolted flange pipe system, the tightness of bolt is directly related to the size and distribution of gasket stress, and is the key factor of joint sealing performance[3]. Bolt strain variation can directly reflect the change of pre-tightening force which directly affects the sealing performance of flange joint. However, in practical engineering applications, it is relatively difficult to directly measure the bolt force. As a result, exploring a method that could indirectly monitor bolt force is of great significance, and this method can also be applied to online monitor the sealing performance of bolted flange joint.

\section{Process of Experiment}

\subsection{Introduction of Experiment}

The incentive applied in this experiment is norm sinusoidal vibration, which refers to the value table of 8 degrees seismic fortification intensity. Considering the actual situation of experiment table, the sizes of vibration acceleration in the $\mathrm{X}$ direction are shown in Table 1.

Table 1 Vibration acceleration in the $\mathrm{X}$ direction

\begin{tabular}{|l|c|c|c|c|c|c|c|c|c|c|}
\hline Frequency /Hz & 2 & 4 & 6.67 & 12.5 & 20 & 40 & 50 & 66.7 & 90.9 & 100 \\
\hline Acceleration /g & 0.5 & 0.7 & 1.0 & 1.0 & 0.8 & 0.6 & 0.56 & 0.52 & 0.49 & 0.48 \\
\hline
\end{tabular}

The sizes of incentive in the $\mathrm{Z}$ direction is the same as the incentive in the $\mathrm{X}$ direction. Theoretically, the sizes of incentive in $\mathrm{Y}$ direction is $65 \%$ of its in the $\mathrm{X}$ direction[4], but the actual sizes of incentive in experiments are shown in Table 2.

Table 2 Vibration acceleration in the $\mathrm{Y}$ direction

\begin{tabular}{|l|c|c|c|c|c|c|c|c|c|c|}
\hline Frequency /Hz & 2 & 4 & 6.67 & 12.5 & 20 & 40 & 50 & 66.7 & 90.9 & 100 \\
\hline Acceleration /g & 0.4 & 0.7 & 0.7 & 0.67 & 0.51 & 0.39 & 0.36 & 0.34 & 0.32 & 0.31 \\
\hline
\end{tabular}

The Experimental test objects are bolt strain and acceleration of pipe flange, which are collected respectively by resistance strain gauge and acceleration sensors. 
Vibration tables are shown in Fig 1\&2:

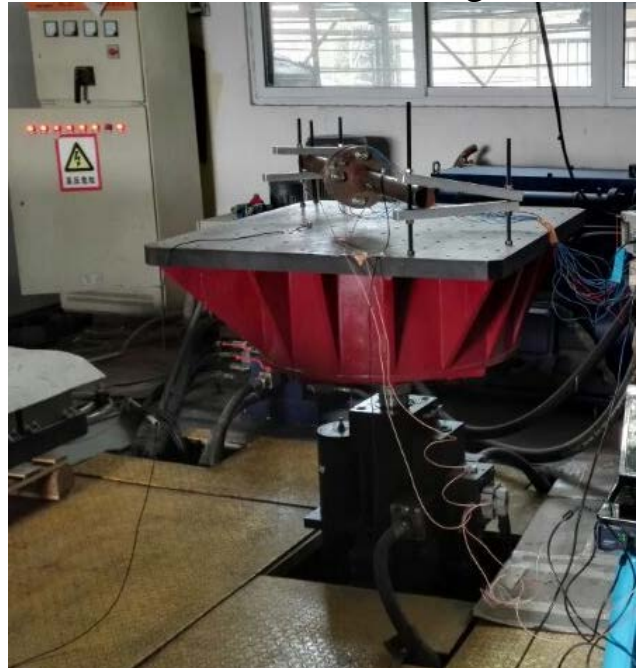

Fig.1 Vertical vibration table

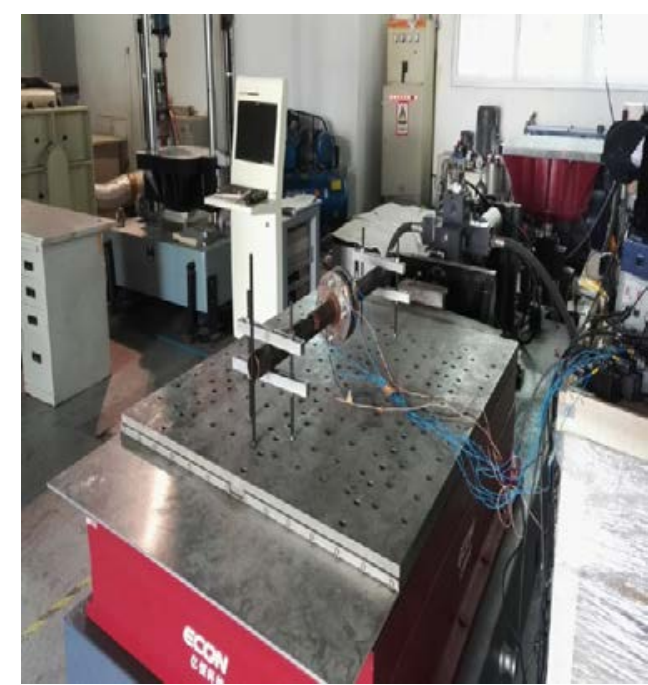

Fig.2 Horizontal vibration table

In order to collect and record signals conveniently, bolts are labeled as 1, 2, 3, 4, which is convenient to tight or loose flange. At the same time, the strain gauge are numbered. During the experiment, four pieces of strain gauge in the No.1 and No.3 bolts are monitored at the same time. Besides, the strain gauge of No.1 bolt are numbered as A1, A2 and the strain gauge of No.3 bolt are numbered as B1, B2.

When vibration incentive is applied, the corresponding bolt pre-tightening load should be applied. In this experiment, the bolt pre-tightening load are respectively F1=10000N, F2=26500N.

\subsection{Experimental Flow Chart}

The experimental flowchart is shown in Fig 3:

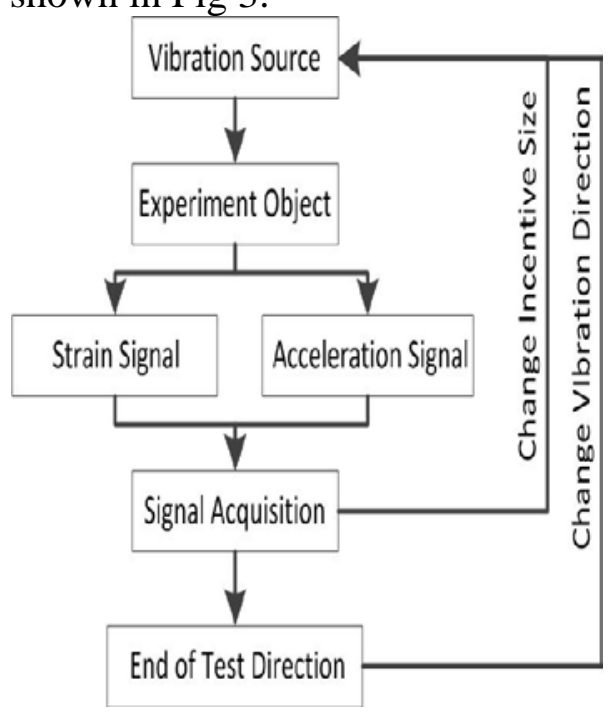

\subsection{Signal Acquisition}

Fig 3. Experimental flow chart

In this experiment, there are two different signals. Strain signals are collected by signal acquisition instrument through the bridge box and acceleration signals are collected by acceleration sensors. the acceleration sensor in the flange is used to measure the acceleration signal in $\mathrm{Z}$ direction. At the same time, in two mutually perpendicular positions which are away from pipe head face for $330 \mathrm{~mm}$ there are two sensors used to measure acceleration signals in $\mathrm{X}$ and $\mathrm{Y}$ directions, which are shown in Fig4. It is very important to make sure that all signals are measured at the same time, so the bridge box and the acceleration sensors are connected to the same signal acquisition instrument. Each experiment is carried out in three directions, and the final results should be compounded by three results in different directions. 


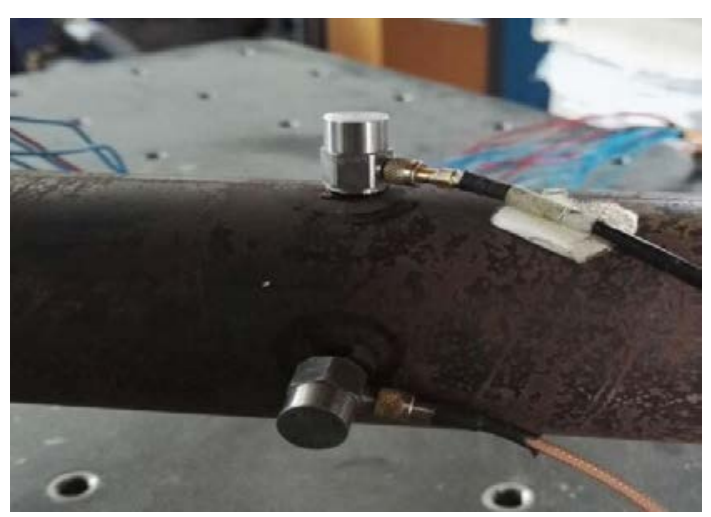

Fig 4. Installing the Sensor

\section{Analysis of Experimental Results}

When the bolted flange system is subjected to vibration, bolt strain will decreases. By data processing, the relationship between strain change and frequency is displayed. When the pre-tightening force of bolts is $\mathrm{F} 1$, considering the vibration in three directions, the relationship between strain change and frequency is shown in Fig5.

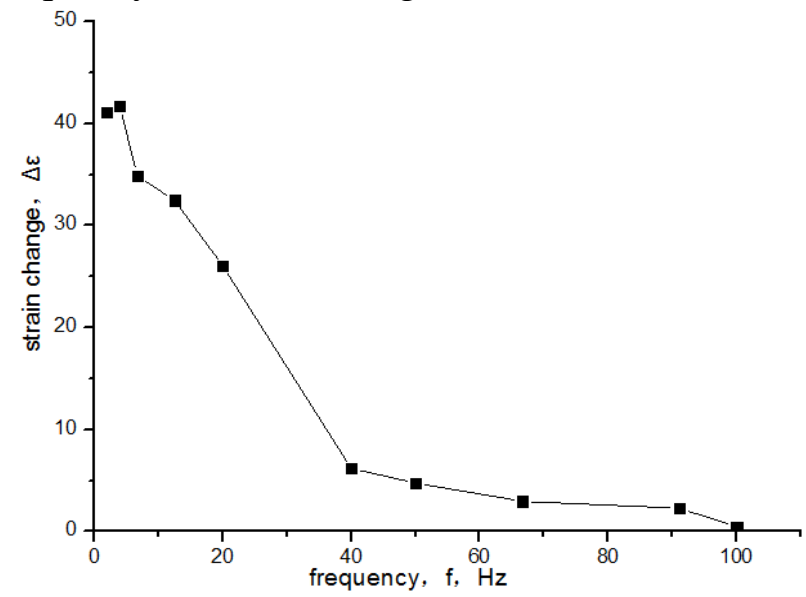

Fig.5 Figure of the total strain change

When the pre-tightening force of bolts is F2, the relationship between strain change and frequency is shown in Fig6.

It was found that under the same vibration condition, the greater of the pre-tightening force is, the smaller of bolt strain is by comparing Fig5 and Fig6.

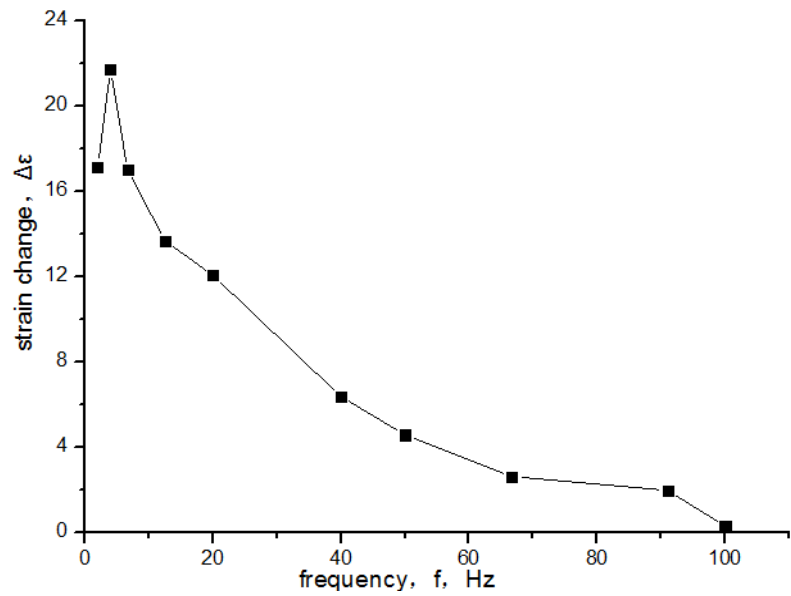

Fig.6 Figure of the total strain change

When the pre-tightening force of bolts is F1, the acceleration of $\mathrm{X}, \mathrm{Y}, \mathrm{Z}$ three directions and bolt strain change in different frequency are shown in Table3. 
Table 3 Acceleration and total strain change

\begin{tabular}{|c|c|c|c|c|c|c|c|c|c|c|}
\hline $\begin{array}{c}\text { Frequency } \\
\text { Case }\end{array}$ & 2 & 4 & 6.67 & 12.5 & 20 & 40 & 50 & 66.7 & 90.9 & 100 \\
\hline X direction & 1.758 & 2.911 & 3.033 & 2.670 & 2.336 & 1.311 & 1.080 & 0.686 & 0.576 & 0.480 \\
\hline Y direction & 1.816 & 2.956 & 2.898 & 2.504 & 1.830 & 1.137 & 0.870 & 0.703 & 0.586 & 0.400 \\
\hline Z direction & 2.109 & 2.864 & 2.858 & 2.511 & 2.252 & 1.479 & 0.820 & 0.499 & 0.506 & 0.396 \\
\hline Total strain & 41.150 & 41.710 & 34.875 & 32.475 & 26.05 & 6.200 & 4.755 & 2.970 & 2.293 & 0.480 \\
\hline
\end{tabular}

The correlation coefficient between acceleration and strain change can be calculated by using the corrcoef function of MATLAB[5,6]. So, the correlation coefficients between acceleration in X, Y, Z directions and strain are $0.8906,0.9205,0.9324$ respectively.

With the same method, when the pre-tightening force is F2, the correlation coefficients between acceleration in X, Y, Z directions and strain are 0.9082, 0.9257, 0.9624 respectively.

The minimum correlation coefficient is 0.8906, and the rest are over 0.9. According to the standards, the acceleration signal and the strain change signal are highly relevant. As a result, in the practical engineering application, the change of acceleration can be used to predict and judge the flange sealing performance.

\section{Equivalent Decision of Sealing Performance}

According to the judging standard of sealing performance, to keep the sealing performance the minimum compression stress (mp) is $18.9 \mathrm{MPa}$. Therefore, the bolt force can be calculated by the minimum compression stress of gaskets.

$$
F_{1}=3.14 D_{G} N m p
$$

$\mathrm{D}_{\mathrm{G}}$ is equal to the average diameter of gasket and $\mathrm{N}$ represents the width of gasket contact surface. The number of bolts is 4 and the calculation formula of single bolt force is following:

$$
F_{2}=\frac{3.14 D_{G} N m p}{4}
$$

By calculating, the single bolt force is $8624 \mathrm{~N}$, which becomes the turning point of the flange sealing performance. When the bolt force is greater than this value, the flange meets the sealing requirements.

When the pre-tightening force is F1 and the bolt force decrease to the value in the front, namely when the decrease reaches to $1376 \mathrm{~N}$, the flange sealing performance will be considered as failure. At that time, the bolt strain change is about $27 \mu \varepsilon$ which can be considered as safety line. Safety line is shown in Fig7.

From the graph above, when the bolt strain change is over $27 \mu \varepsilon$, the flange sealing performance can be considered dangerous and more likely to leak.

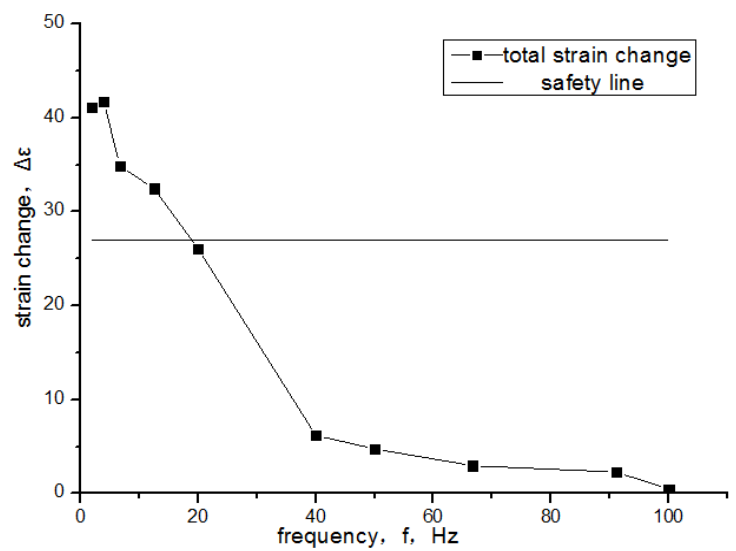

Fig.7 Strain change and safety line

According to the previous correlation analysis, it shows great correlation between the 
acceleration and strain change. According to this conclusion, Fig7 can be transformed to Fig8, which shows the acceleration in $\mathrm{X}$ direction and the safety line. In a similar way, the figures which show the acceleration in $\mathrm{Y}, \mathrm{Z}$ direction and the safety line can be obtained.

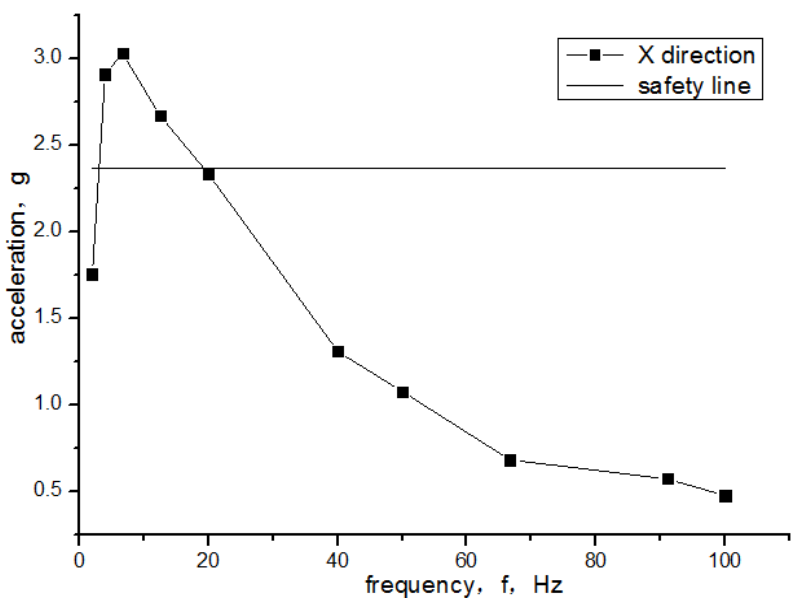

Fig.8 Acceleration and safety line

When the pre-tightening force is F2 and the bolt force decreases to the critical value, namely when the decrease is $17876 \mathrm{~N}$, the bolt strain change is about $346.7 \mu \varepsilon$. According to the figure 8, in the process of vibration, the maximum strain change of the bolt is $21.725 \mu \varepsilon$, far less than $346.7 \mu \varepsilon$. So the bolt is in a safe range and the sealing performance of flange joint is up to standard.

\section{Conclusion}

Bolt strain variation and the change of pipe acceleration in three directions were recorded when different vibration excitation is applied in experimental subjects. Then this experiment was repeated by changing different bolt force. Experimental results show that:

* The increase of bolt force can improve the sealing performance of bolted flange joint in vibration environment. There are two main reasons: firstly, the increase of bolt force rises the difference value of failure critical value, so the bolt strain change of seal failure increases. Secondly, in the process of vibration, the increase of bolt force makes the bolt strain change smaller under the same vibration condition;

* It was found that the acceleration signal and the strain change signal are highly relevant. Therefore, the change of bolt force can be characterized by the change of acceleration and this method can also provide certain theoretical basis for online monitoring the sealing performance of bolted flange joint.

\section{Acknowledgements}

This work is supported by National Science Foundation of China (No. 51275171).

\section{References}

[1]Dario Di Maio ,Christoph Schwingshackl,Ibrahim A. Sever. Development of A Test Planning Methodology for Performing Experimental Model Validation of Bolted Flanges[J]. Nonlinear Dynamics.Vol.83(2016) :p.983-1002.

[2]Oreste S. Bursia, Md S. Rezaa, Giuseppe Abbiatia, Fabrizio Paolacci.Performance-based earthquake evaluation of a full-scale petrochemical piping system[J].Loss Prevention in the Process Industries.Vol.33(2015):p.10-22.

[3]XU Tao-long,YAO An-lin,JIANG Hong-ye,et al.Key Techniques of Risk Assessment of Oil and Gas Pipelines Damaged by third party[J]. Journal of Oil and Gas Technology.Vol.33(2011) 
NO.2,p.150-154.

[4]WANG Xian—nong,ZHOU Xiao-hui,ZHANG Fu—bin.Computational analysis of steel frame structures earthquake spectrum action by using ANSYS[J].Journal of Hebei University of Engineering.Vol.30(2013) NO.3.

[5]Dalun Wang,et al. The Processing of Digital Signal-Theory and Practice.Tsinghua University Press ,2010.

[6]Guochao Li,et al. The Fundamentals and Applications of MATLAB. Peking University Press,2011. 